\title{
FRESH FACES FOR ESTABLISHED BRANDS: THE ROLE OF HOLISTIC TYPE AND SEQUENCE OF EXPOSURE IN CONSUMERS' FORMATION OF TRAIT INFERENCES
}

\author{
Yonca Limon, Christian-Albrechts-University Kiel, Germany \\ Martin Reimann, USC, U.S.A. \\ Ulrich R. Orth, Christian-Albrechts-University Kiel, Germany \\ Antoine Bechara, USC, U.S.A.
}

\begin{abstract}
Firms frequently select endorsers on the basis of specific physical characteristics, such as the face, to better differentiate and position their brands. While some research exists that aids managers in selecting human faces, several gaps call for closing. Those gaps include existing insights into effects for only a few face types (e.g., 'baby face', 'classical beauty'), primarily new (rather than established) brands, celebrity endorsers (rather then typical consumers), simultaneous (rather then sequential) exposure of consumers to both faces and brands, and a focus on cognitive rather than emotional processes.

Five studies aim to close these gaps in prior research by investigating the roles of holistic types of endorser faces and mode of exposure (simultaneous vs. sequential presentation of face and brand) in the generation of brand personality impressions that guide consumer behavior. Studies 1 and 2 provide evidence that consumers develop brand personality impressions from trait inferences of endorsers' holistic face types rather than single anatomical features. Studies 3 and 4 show that the match or mismatch between face-based and brand-based trait inferences as well as the modes of exposure (simultaneous vs. sequential presentation of face and brand) affect brand impressions and behavioral intentions. Study 5 shows that matching endorser face and brand on salient personality traits as well as presenting them simultaneously create a unique emotional basis that leads to increased brand choice. Implications are discussed for advertising practice and research.
\end{abstract}

References available upon request 